\title{
A Derivation of Stored Electromagnetic Field Energies in an Arbitrary Medium
}

This paper was downloaded from TechRxiv (https://www.techrxiv.org).

\section{LICENSE}

CC BY 4.0

SUBMISSION DATE / POSTED DATE

28-02-2022 / 03-03-2022

\section{CITATION}

Geyi, Wen (2022): A Derivation of Stored Electromagnetic Field Energies in an Arbitrary Medium. TechRxiv. Preprint. https://doi.org/10.36227/techrxiv.19248254.v1

DOI

10.36227/techrxiv.19248254.v1 


\title{
A Derivation of Stored Electromagnetic Field Energies in an Arbitrary Medium
}

\author{
Wen Geyi, Fellow, IEEE
}

\begin{abstract}
The stored energies of time-harmonic field in a medium are traditionally analyzed by using narrow-band (NB) approximation. The analysis, however, suffers from two problems. Firstly, it involves a specific constitutive relation and is thus only applicable to a specified medium. Secondly, it is incompatible with the time-harmonic field theory for the stored energy expressions derived from it include time-dependent complex envelops rather than phasors. This article aims to solve the problems with the traditional NB analysis. The expressions of the stored energies in an arbitrary medium have been derived by introducing a new NB technique, which is based on the understanding that a pure sinusoidal field may be viewed as the limit of a NB field as its bandwidth shrinks to zero. To facilitate the derivation, an explicit form of the complex envelope is first deduced by expanding the Fourier frequency spectrum of the NB field around the mid-band frequency. The inflow power from the external source can be divided into the sum of the rate of dissipated energy and the rate of increase of stored energy. After introducing the time-domain fields derived from the new NB approach, the rates of dissipated energies and the stored energies can be determined.
\end{abstract}

Index Terms-Wave propagation in medium, stored field energy, dissipated field energy.

\section{INTRODUCTION}

The stored electromagnetic (EM) field energies are important quantities in the study of wave propagation and energy storage in electrical and optical engineering, and many efforts have been placed on their evaluation in various media [1]-[23]. In the study of the stored energy expressions for a sinusoidal (time-harmonic) field, a narrow-band (NB) analysis is traditionally utilized by assuming that the field has frequency components in a narrow range about some central value $\omega_{0}[2]-[7],[24]$

$$
\mathbf{E}(t)=\operatorname{Re} \mathbf{E}_{a}(t) e^{j \omega_{0} t},
$$

where $\mathbf{E}_{a}(t)$ is referred to as the complex envelope and is supposed to be a slowly varying function of time compared with the carrier wave $e^{j \omega_{0} t}$, and $\omega_{0}$ is the mid-band frequency, also called the carrier frequency. The complex envelope $\mathbf{E}_{a}(t)$ reduces to the conventional phasor for a pure sinusoidal field if it is independent of time.

The traditional NB analysis for stored field energies suffers from two major drawbacks[2]-[7]. Firstly, a specific constitutive relation has to be assumed, which imposes a restriction on the generality of the derived energy expressions.

This work was supported by the National Natural Science Foundation of China under Grant 61971231. The author is with Research Center of Applied Electromagnetics, Nanjing University of Information Science and Technology, Nanjing 210044, China (e-mail: wgy@nuist.edu.cn).
Secondly, the derived energy expressions include timedependent complex envelopes and are thus incompatible with the conventional time-harmonic field theory where the time-independent phasors are commonly used.

In this article, the stored EM field energies in an arbitrary medium will be investigated by introducing a new NB approach, the basic idea behind which is to treat the sinusoidal field as the limit of a NB field when its bandwidth tends to zero. In order to solve the above-mentioned problems with the traditional NB analysis, the NB field is first analyzed in Fourier frequency domain. By means of the expansion of the Fourier frequency spectrum around the mid-band frequency, explicit expressions for the complex envelopes of the NB field can be found. When the complex envelopes are introduced into the time-domain Poynting theorem, the energy expressions, which are valid for an arbitrary medium and expressed in terms of phasors, can be identified.

\section{TRADITIONAL NARROW-BAND APPROACH}

For completeness and clarity, we will briefly review the traditional NB analysis for studying the stored field energies for a time-harmonic field. The analysis is usually based on the time-domain Poynting theorem in a source-free region

$$
-\nabla \cdot[\mathbf{E}(t) \times \mathbf{H}(t)]=\mathbf{E}(t) \cdot \frac{\partial \mathbf{D}(t)}{\partial t}+\mathbf{H}(t) \cdot \frac{\partial \mathbf{B}(t)}{\partial t},
$$

where all symbols have their conventional meanings in EM theory. To simplify the notations, we will use $\mathbf{F}(\omega)$ to denote the phasor of a sinusoidal vector field, and $\mathbf{F}(t)$ the same vector field in time domain without explicitly showing the space dependence. The left-hand side of (2) represents the inflow power density from the external source, and consequently the right-hand side of (2) may be interpreted as the sum of the rate of the dissipated field energy density and the rate of increase of the total stored field energy density. As mentioned in [6], it is difficult to interpret the right-hand side as the time derivative of an instantaneous energy density in a general medium. Physically, the right-hand side of (2) consists of two terms respectively related to the electric and magnetic fields, each of which may be decomposed into two parts. One part is converted into heat loss, and the other is stored in the medium and is recoverable in the form of EM field energy, as described below

$$
\begin{aligned}
& \mathbf{E}(t) \cdot \frac{\partial \mathbf{D}(t)}{\partial t}=p_{e}(t)+\frac{d w_{e}(t)}{d t}, \\
& \mathbf{H}(t) \cdot \frac{\partial \mathbf{B}(t)}{\partial t}=p_{m}(t)+\frac{d w_{m}(t)}{d t},
\end{aligned}
$$

where $p_{e}(t)$ and $p_{m}(t)$ denote the rates of dissipated electric and magnetic field energy density, respectively; $w_{e}(t)$ and 
$w_{m}(t)$ stand for the stored electric and magnetic field energy densities, respectively. The above decompositions can be easily understood from the point of view of circuit theory [1].

In the traditional NB approach, an assumption is often made that the medium is dispersive and has negligible losses [2]-[7]. By means of the NB representation (1), the left-hand side of (3) can be written as

$$
\mathbf{E} \cdot \frac{\partial \mathbf{D}}{\partial t}=\frac{1}{4}\left(\mathbf{E}_{a} e^{j \omega_{0} t}+\overline{\mathbf{E}}_{a} e^{-j \omega_{0} t}\right) \cdot \frac{\partial}{\partial t}\left(\mathbf{D}_{a} e^{j \omega_{0} t}+\overline{\mathbf{D}}_{a} e^{-j \omega_{0} t}\right)
$$

where the overbar denotes the complex conjugate. The complex envelope $\mathbf{E}_{a}$ is then expanded as an integral of the Fourier components $\tilde{\mathbf{E}}_{a} e^{j \omega t}$, where $\tilde{\mathbf{E}}_{a}$ is independent of time. According to the NB assumption, the Fourier frequency components must satisfy $\omega \ll \omega_{0}$ to ensure that the complex envelope varies slowly. A specific constitutive relation such as $\mathbf{D}=\varepsilon \mathbf{E}$, where $\varepsilon$ is the dielectric constant and is real for a lossless medium, can be introduced, and we thus have the following component-wise expression

$$
\begin{array}{r}
\frac{\partial \mathbf{D}}{\partial t}=\frac{\partial}{\partial t}\left(\tilde{\mathbf{D}}_{a} e^{j\left(\omega+\omega_{0}\right) t}\right)=j\left(\omega+\omega_{0}\right) \tilde{\mathbf{D}}_{a} e^{j\left(\omega+\omega_{0}\right) t} \\
=j\left(\omega+\omega_{0}\right) \varepsilon\left(\omega+\omega_{0}\right) \tilde{\mathbf{E}}_{a} e^{j\left(\omega+\omega_{0}\right) t} .
\end{array}
$$

Upon expanding the factor $\left(\omega+\omega_{0}\right) \varepsilon\left(\omega+\omega_{0}\right)$ about the carrier frequency $\omega_{0}$ up to the linear term (first-order approximation), the above expression can be written as

$$
\frac{\partial \mathbf{D}}{\partial t}=j \omega_{0} \varepsilon\left(\omega_{0}\right) \tilde{\mathbf{E}}_{a} e^{j\left(\omega+\omega_{0}\right) t}+j \omega \frac{d\left[\omega_{0} \varepsilon\left(\omega_{0}\right)\right]}{d \omega_{0}} \tilde{\mathbf{E}}_{a} e^{j\left(\omega+\omega_{0}\right) t}
$$

Summing the Fourier components yields

$$
\frac{\partial \mathbf{D}}{\partial t}=j \omega_{0} \varepsilon\left(\omega_{0}\right) \mathbf{E}_{a} e^{j \omega_{0} t}+\frac{d\left[\omega_{0} \varepsilon\left(\omega_{0}\right)\right]}{d \omega_{0}} \frac{\partial \mathbf{E}_{a}}{\partial t} e^{j \omega_{0} t}
$$

Substituting (6) into (5), we obtain

$$
\begin{array}{r}
\mathbf{E} \cdot \frac{\partial \mathbf{D}}{\partial t}=\frac{1}{4} \frac{d\left[\omega_{0} \varepsilon\left(\omega_{0}\right)\right]}{d \omega_{0}} \mathbf{E}_{a} \frac{\partial \overline{\mathbf{E}}_{a}}{\partial t}+\frac{1}{4} \frac{d\left[\omega_{0} \varepsilon\left(\omega_{0}\right)\right]}{d \omega_{0}} \overline{\mathbf{E}}_{a} \frac{\partial \mathbf{E}_{a}}{\partial t} \\
+ \text { terms containing } e^{ \pm j 2 \omega_{0} t} \\
=\frac{\partial}{\partial t}\left[\frac{1}{4} \frac{d\left[\omega_{0} \varepsilon\left(\omega_{0}\right)\right]}{d \omega_{0}}\left|\mathbf{E}_{a}\right|^{2}\right]+\text { terms containing } e^{ \pm j 2 \omega_{0} t} .
\end{array}
$$

The terms containing $e^{ \pm j 2 \omega_{0} t}$ can be ignored since they are approximately zero after the time average over one period of the carrier wave. Comparing (7) with (3), we may find the stored electric field energy in time domain

$$
w_{e}(t)=\frac{1}{4} \frac{d\left[\omega_{0} \varepsilon\left(\omega_{0}\right)\right]}{d \omega_{0}}\left|\mathbf{E}_{a}\right|^{2}
$$

Once this is averaged over one period of the carrier wave, we obtain the stored electric field energy in frequency domain

$$
w_{e}\left(\omega_{0}\right)=\frac{1}{T_{0}} \int_{0}^{T_{0}} w_{e}(t) d t=\frac{1}{4} \frac{d\left[\omega_{0} \varepsilon\left(\omega_{0}\right)\right]}{d \omega_{0}}\left|\mathbf{E}_{a}\right|^{2},
$$

where the complex envelope $\mathbf{E}_{a}$ is assumed to be constant over $\left[0, T_{0}\right]$ with $T_{0}=2 \pi / \omega_{0}$ representing the period of the carrier wave. As pointed out in [6], the complex envelope $\mathbf{E}_{a}$ in (8) is still considered to be time-dependent. An expression for the magnetic field in a medium with $\mathbf{B}=\mu \mathbf{H}$ can be found in a similar way

$$
w_{m}\left(\omega_{0}\right)=\frac{1}{4} \frac{d\left[\omega_{0} \mu\left(\omega_{0}\right)\right]}{d \omega_{0}}\left|\mathbf{H}_{a}\right|^{2}
$$

where $\mu$ is the permeability of the medium.

The above discussion occurs frequently in textbooks (e.g., [2]-[7]) and has been a typical procedure to find the stored energy expressions in a dispersive medium. There are two problems with the above procedure that has limited the applicability of the traditional NB approach. One of them is that a specific constitutive relation (i.e., medium parameters $\mu$ and $\varepsilon$ in the above) has to be introduced and expanded around the carrier frequency, and the energy expressions (8) and (9) are therefore medium specific. The other is that the time-averaged expressions (8) and (9) involve the complex envelopes $\mathbf{E}_{a}$ and $\mathbf{H}_{a}$, instead of the phasors commonly used for a sinusoidal field. For this reason, the energy expressions (8) and (9) are incompatible with the time-harmonic field theory since the complex envelopes weakly depend on time while the phasors do not. In what follows, a new NB approach will be developed to solve these problems.

\section{AN EXPLICIT EXPRESSION FOR THE COMPLEX ENVELOPE}

Consider the Fourier transform pair

$$
\mathbf{E}(t)=\frac{1}{2 \pi} \int_{-\infty}^{\infty} \tilde{\mathbf{E}}(\omega) e^{j \omega t} d \omega, \tilde{\mathbf{E}}(\omega)=\int_{-\infty}^{\infty} \mathbf{E}(t) e^{-j \omega t} d t,
$$

where $\tilde{\mathbf{E}}(\omega)$ is the Fourier frequency spectrum (FFS) of the time-domain electric field $\mathbf{E}(t)$. Since $\mathbf{E}(t)$ is a real function of time, we have the following symmetry property of the FFS about the frequency

$$
\tilde{\mathbf{E}}(-\omega)=\overline{\tilde{\mathbf{E}}}(\omega)
$$

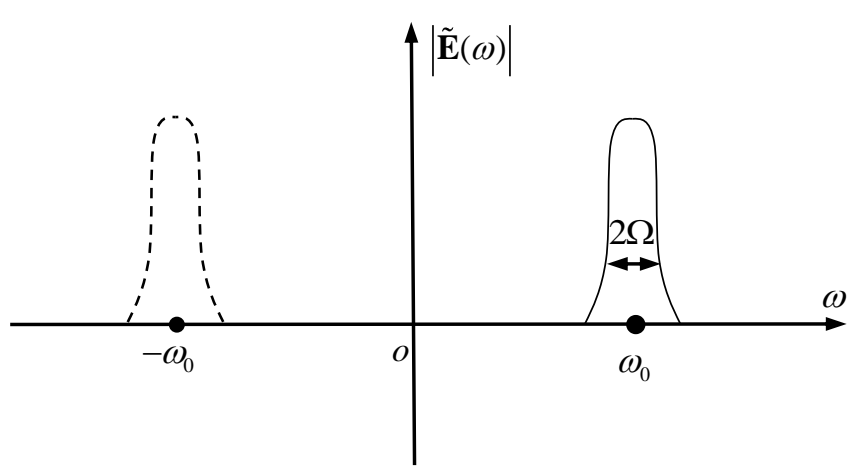

Figure 1 FSS of NB vector field.

The electric field $\mathbf{E}(t)$ can thus be expressed in terms of FFS as an integral along the positive frequency axis

$$
\mathbf{E}(t)=\operatorname{Re} \frac{1}{\pi} \int_{0}^{\infty} \tilde{\mathbf{E}}(\omega) e^{j \omega t} d \omega .
$$

If the FFS of the electric field $\mathbf{E}(t)$ is narrow banded and centered at a mid-band frequency $\omega_{0}$ and confined in the narrow range $\left( \pm \omega_{0}-\Omega, \pm \omega_{0}+\Omega\right)$ with sufficiently small half-bandwidth $\Omega$ (see Figure 1), the integrand in (12) may be considered as a constant in the narrow frequency range 
(zeroth-order approximation) and can therefore be approximated by its value at the central frequency $\omega_{0}$. As a result, the electric field (12) can be written as

$$
\mathbf{E}(t)=\operatorname{Re} \frac{1}{\pi} \tilde{\mathbf{E}}\left(\omega_{0}\right) e^{j \omega_{0} t} \int_{\omega_{0}-\Omega}^{\omega_{0}+\Omega} d \omega=\operatorname{Re} \mathbf{E}\left(\omega_{0}\right) e^{j \omega_{0} t},
$$

where

$$
\mathbf{E}\left(\omega_{0}\right)=\frac{2 \Omega}{\pi} \tilde{\mathbf{E}}\left(\omega_{0}\right)
$$

is the complex envelope for the NB electric field $\mathbf{E}(t)$ and is independent of time. When the half-bandwidth $\Omega$ shrinks to zero, $\tilde{\mathbf{E}}\left(\omega_{0}\right)$ approaches to infinity but the complex envelope (14) remains finite for the reason that the electric field $\mathbf{E}(t)$ must be bounded. In the limit of $\Omega \rightarrow 0$, (13) turns out to be a pure sinusoidal wave and the complex envelope (14) becomes the conventional phasor in time-harmonic field theory.

For the study of the stored field energies, one needs to consider the time derivative of the field involved in the quantity $\mathbf{E} \cdot \partial \mathbf{D} / \partial t$, which has to be decomposed according to (3). For this reason, the zeroth-order approximation (13) for the NB electric field is no longer accurate enough and the time variation of the complex envelope must be taken into account. In this case, one has to resort to the first-order approximation for the FFS of the NB field around the mid-band frequency

$$
\tilde{\mathbf{E}}(\omega) \approx \tilde{\mathbf{E}}\left(\omega_{0}\right)+\left(\omega-\omega_{0}\right) \frac{\partial \tilde{\mathbf{E}}\left(\omega_{0}\right)}{\partial \omega} .
$$

Introducing the expansion (15) into the Fourier integral (12), the NB electric field can be expressed by

$$
\begin{aligned}
\mathbf{E}(t) & =\frac{1}{\pi} \operatorname{Re}\left[\tilde{\mathbf{E}}\left(\omega_{0}\right) \int_{\omega_{0}-\Omega}^{\omega_{0}+\Omega} e^{j \omega t} d \omega\right] \\
& +\frac{1}{\pi} \operatorname{Re}\left[\frac{\partial \tilde{\mathbf{E}}\left(\omega_{0}\right)}{\partial \omega} \int_{\omega_{0}-\Omega}^{\omega_{0}+\Omega}\left(\omega-\omega_{0}\right) e^{j \omega t} d \omega\right] .
\end{aligned}
$$

By means of the following calculations

$$
\begin{aligned}
& \int_{\omega_{0}-\Omega}^{\omega_{0}+\Omega} e^{j \omega t} d \omega=\frac{2 \sin \Omega t}{t} e^{j \omega_{0} t} \\
& \int_{\omega_{0}-\Omega}^{\omega_{0}+\Omega}\left(\omega-\omega_{0}\right) e^{j \omega t} d \omega=-j e^{j \omega_{0} t} \frac{d}{d t}\left(\frac{2 \sin \Omega t}{t}\right),
\end{aligned}
$$

the time-domain expression (16) can be expressed in the conventional NB form

$$
\mathbf{E}(t)=\operatorname{Re} \mathbf{E}_{a}(t) e^{j \omega_{0} t},
$$

where the complex envelope is

$$
\mathbf{E}_{a}(t)=\tilde{\mathbf{E}}\left(\omega_{0}\right) g(t)-j \frac{\partial \tilde{\mathbf{E}}\left(\omega_{0}\right)}{\partial \omega} g^{\prime}(t)
$$

with $g(t)$ and $g^{\prime}(t)$ respectively given by

$$
g(t)=\frac{2 \Omega}{\pi} \frac{\sin \Omega t}{\Omega t}, g^{\prime}(t)=\Omega \frac{2 \Omega}{\pi} \frac{\Omega t \cos \Omega t-\sin \Omega t}{(\Omega t)^{2}} .
$$

The complex envelope (18) is composed of two terms and is more informative than just being slowly varying function of time assumed a priori in the traditional NB analysis. The first term $\mathbf{E}\left(\omega_{0}\right) g(t)$ becomes the complex envelope (14) or the conventional phasor for a sinusoidal field as $\Omega \rightarrow 0$, while the second term contains the frequency derivative of the field and tends to zero as $\Omega \rightarrow 0$. It is noted that the traditional NB analysis only uses the first-order approximation for the medium parameters and has ignored the frequency derivatives of the fields. For this reason, the energy expressions derived from it are less accurate, and are only valid for the specified medium parameters.

The FFS of the NB field (17) can be written as

$$
\tilde{\mathbf{E}}(\omega)=\frac{1}{2}\left[\tilde{\mathbf{E}}_{a}\left(\omega-\omega_{0}\right)+\overline{\tilde{\mathbf{E}}}_{a}\left(-\omega-\omega_{0}\right)\right],
$$

where $\tilde{\mathbf{E}}_{a}(\omega)$ is the FFS of the complex envelope $\mathbf{E}_{a}(t)$. Clearly, the FFS $\tilde{\mathbf{E}}(\omega)$ is the sum of two shifted versions of $\tilde{\mathbf{E}}_{a}(\omega)$, which are centered at $\pm \omega_{0}$, as sketched in Figure 1.

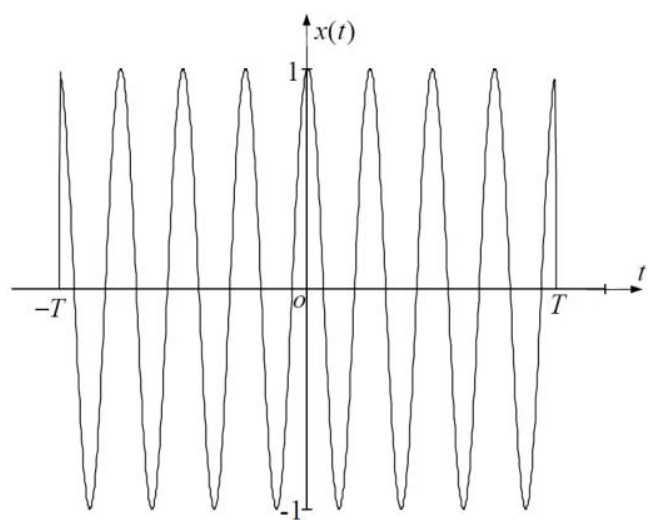

(a)

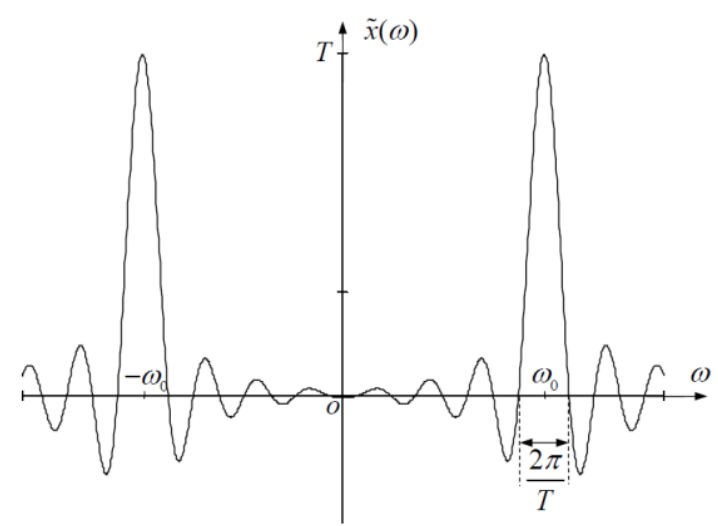

(b)

Figure 2 (a) PMSS. (b) FFS.

To show how a pure sinusoidal wave can be viewed as the limit of a NB wave as its bandwidth shrinks to zero, let us consider a pulse-modulated sinusoidal signal (PMSS, a truncated sinusoidal wave) shown in Figure 2(a)

$$
x(t)=\operatorname{Re} p_{T}(t) e^{j \omega_{0} t},
$$

where the envelope $p_{T}(t)$ is a rectangular pulse of width $2 T$ defined by

$$
p_{T}(t)=\left\{\begin{array}{ll}
0, & |t|>0 \\
1, & |t|<T
\end{array} .\right.
$$

The FFS of the envelope is a sinc pulse, and the FFS of the 
PMSS (21) consists of two sinc pulses centered at $\omega= \pm \omega_{0}$ with peak value $T$ and width $2 \Omega=2 \pi / T$ as depicted in Figure 2(b). Explicitly the FFS of the PMSS is given by [28]

$$
\tilde{x}(\omega)=T \frac{\sin \left(\omega-\omega_{0}\right) T}{\left(\omega-\omega_{0}\right) T}+T \frac{\sin \left(\omega+\omega_{0}\right) T}{\left(\omega+\omega_{0}\right) T} .
$$

As the half-duration $T$ of the rectangular pulse increases, the FFS $\tilde{x}(\omega)$ becomes more concentrated about $\pm \omega_{0}$ but the product of the peak value $T$ of the two sinc pulses and their width $2 \Omega=2 \pi / T$ remains constant. In the limit of the half-duration $T \rightarrow \infty$ or the half-bandwidth $\Omega \rightarrow 0$, the PMSS approaches to a pure sinusoidal wave and its FFS (22) becomes the sum of two delta functions centered at $\pm \omega_{0}$

$$
\tilde{x}(\omega)=\pi\left[\delta\left(\omega-\omega_{0}\right)+\delta\left(\omega+\omega_{0}\right)\right] .
$$

\section{STORED FIELD ENERGIES FOR TIME-HARMONIC FIELD}

In order to find the stored electric field energy and the rate of the dissipated electric field energy, we need to split $\mathbf{E} \cdot \partial \mathbf{D} / \partial t$ according to (3). Making use of the NB representation (17) for the electric field $\mathbf{E}$ and a similar representation for the electric induction intensity $\mathbf{D}$, the left-hand side of (3) can be written as

$$
\begin{array}{r}
\mathbf{E}(t) \cdot \frac{\partial \mathbf{D}(t)}{\partial t}= \\
\frac{1}{4}\left\{\tilde{\mathbf{E}}\left(\omega_{0}\right) g(t)-j \frac{\partial \tilde{\mathbf{E}}\left(\omega_{0}\right)}{\partial \omega} g^{\prime}(t)\right\} \cdot\left\{\tilde{\tilde{\mathbf{D}}}\left(\omega_{0}\right) g^{\prime}(t)+j \frac{\partial \overline{\tilde{\mathbf{D}}}\left(\omega_{0}\right)}{\partial \omega} g^{\prime \prime}(t)\right\} \\
-\frac{j \omega_{0}}{4}\left\{\tilde{\mathbf{E}}\left(\omega_{0}\right) g(t)-j \frac{\partial \tilde{\mathbf{E}}\left(\omega_{0}\right)}{\partial \omega} g^{\prime}(t)\right\} \cdot\left\{\overline{\tilde{\mathbf{D}}}\left(\omega_{0}\right) g(t)+j \frac{\partial \overline{\tilde{\mathbf{D}}}\left(\omega_{0}\right)}{\partial \omega} g^{\prime}(t)\right\} \\
+\frac{1}{4}\left\{\overline{\tilde{\mathbf{E}}}\left(\omega_{0}\right) g(t)+j \frac{\partial \tilde{\tilde{\mathbf{E}}}\left(\omega_{0}\right)}{\partial \omega} g^{\prime}(t)\right\} \cdot\left\{\tilde{\mathbf{D}}\left(\omega_{0}\right) g^{\prime}(t)-j \frac{\partial \tilde{\mathbf{D}}\left(\omega_{0}\right)}{\partial \omega} g^{\prime \prime}(t)\right\} \\
+\frac{j \omega_{0}}{4}\left\{\overline{\tilde{\mathbf{E}}}\left(\omega_{0}\right) g(t)+j \frac{\partial \overline{\tilde{\mathbf{E}}}\left(\omega_{0}\right)}{\partial \omega} g^{\prime}(t)\right\} \cdot\left\{\tilde{\mathbf{D}}\left(\omega_{0}\right) g(t)-j \frac{\partial \tilde{\mathbf{D}}\left(\omega_{0}\right)}{\partial \omega} g^{\prime}(t)\right\} \\
+ \text { terms containing } e^{ \pm j 2 \omega_{0} t} .
\end{array}
$$

Since all quantities will be averaged over one period of the carrier wave $e^{j \omega_{0} t}$ eventually, the terms containing $e^{ \pm j 2 \omega_{0} t}$ can be ignored as they will vanish after the time average. After expanding the right-hand side of the above equation and rearranging the terms, we obtain

$$
\begin{array}{r}
\mathbf{E}(t) \cdot \frac{\partial \mathbf{D}(t)}{\partial t}= \\
\frac{1}{4} \frac{\partial}{\partial t} \operatorname{Re}\left[\tilde{\mathbf{E}}\left(\omega_{0}\right) \cdot \overline{\tilde{\mathbf{D}}}\left(\omega_{0}\right) g^{2}(t)\right]+\frac{1}{4} \frac{\partial}{\partial t} \operatorname{Re} \omega_{0}\left[\tilde{\mathbf{E}}\left(\omega_{0}\right) \cdot \frac{\partial \overline{\tilde{\mathbf{D}}}\left(\omega_{0}\right)}{\partial \omega} g^{2}(t)\right] \\
-\frac{1}{4} \frac{\partial}{\partial t} \operatorname{Re}\left[\omega_{0} \overline{\tilde{\mathbf{D}}}\left(\omega_{0}\right) \cdot \frac{\partial \tilde{\mathbf{E}}\left(\omega_{0}\right)}{\partial \omega} g^{2}(t)\right]+\frac{1}{2} \omega_{0} \operatorname{Im}\left[\tilde{\mathbf{E}}\left(\omega_{0}\right) \cdot \overline{\tilde{\mathbf{D}}}\left(\omega_{0}\right) g^{2}(t)\right] \\
-\frac{1}{2} \operatorname{Im}\left[\tilde{\mathbf{D}}\left(\omega_{0}\right) \cdot \frac{\partial \overline{\tilde{\mathbf{E}}}\left(\omega_{0}\right)}{\partial \omega} g^{\prime}(t) g^{\prime}(t)\right]-\frac{1}{2} \omega_{0} \operatorname{Im}\left[\frac{\partial \overline{\tilde{\mathbf{E}}}\left(\omega_{0}\right)}{\partial \omega} \frac{\partial \tilde{\mathbf{D}}\left(\omega_{0}\right)}{\partial \omega} g^{\prime}(t) g^{\prime}(t)\right] \\
+\frac{1}{2} \operatorname{Re}\left[\frac{\partial \tilde{\mathbf{E}}\left(\omega_{0}\right)}{\partial \omega} \cdot \frac{\partial \overline{\tilde{\mathbf{D}}}\left(\omega_{0}\right)}{\partial \omega} g^{\prime}(t) g^{\prime \prime}(t)\right]-\frac{1}{2} \operatorname{Im}\left[\tilde{\mathbf{E}}\left(\omega_{0}\right) \cdot \frac{\partial \overline{\tilde{\mathbf{D}}}\left(\omega_{0}\right)}{\partial \omega} g(t) g^{\prime \prime}(t)\right] .
\end{array}
$$

where the second derivative of $g(t)$ is given by

$$
g^{\prime \prime}(t)=\frac{2 \Omega}{\pi} \Omega^{3} t \frac{-(\Omega t)^{2} \sin \Omega t-2(\Omega t \cos \Omega t-\sin \Omega t)}{(\Omega t)^{4}} .
$$

As $\Omega \rightarrow 0$, the following asymptotic relations can be easily obtained from (19) and (25) for a finite time $t$

$$
\begin{aligned}
& g(t) \approx \frac{2 \Omega}{\pi} \propto \Omega, \\
& g^{\prime}(t) \approx-\frac{2 \Omega^{3} t}{3 \pi} \propto \Omega^{2}, \\
& g^{\prime \prime}(t) \approx-\frac{2 \Omega^{3}}{3 \pi} \propto \Omega^{3} .
\end{aligned}
$$

As a result, the terms containing $g^{\prime}(t) g^{\prime}(t), g(t) g^{\prime \prime}(t)$ and $g^{\prime}(t) g^{\prime \prime}(t)$ in (24) will disappear as $\Omega \rightarrow 0$ while the terms containing $g^{2}(t)$ in (24) remain finite and become

$$
\begin{aligned}
& \tilde{\mathbf{E}}\left(\omega_{0}\right) \cdot \overline{\tilde{\mathbf{D}}}\left(\omega_{0}\right) g^{2}(t) \rightarrow \mathbf{E}\left(\omega_{0}\right) \cdot \overline{\mathbf{D}}\left(\omega_{0}\right), \\
& \tilde{\mathbf{E}}\left(\omega_{0}\right) \cdot \frac{\partial \overline{\mathbf{D}}\left(\omega_{0}\right)}{\partial \omega} g^{2}(t) \rightarrow \mathbf{E}\left(\omega_{0}\right) \cdot \frac{\partial \overline{\mathbf{D}}\left(\omega_{0}\right)}{\partial \omega}, \\
& \overline{\tilde{\mathbf{D}}}\left(\omega_{0}\right) \cdot \frac{\partial \tilde{\mathbf{E}}\left(\omega_{0}\right)}{\partial \omega} g^{2}(t) \rightarrow \overline{\mathbf{D}}\left(\omega_{0}\right) \cdot \frac{\partial \mathbf{E}\left(\omega_{0}\right)}{\partial \omega},
\end{aligned}
$$

where $\mathbf{E}\left(\omega_{0}\right)=\frac{2 \Omega}{\pi} \tilde{\mathbf{E}}\left(\omega_{0}\right)$ and $\mathbf{D}\left(\omega_{0}\right)=\frac{2 \Omega}{\pi} \tilde{\mathbf{D}}\left(\omega_{0}\right)$ are the conventional phasors defined by (14) when $\Omega \rightarrow 0$. Taking the above relations into account, (24) can be rewritten as

$$
\begin{array}{r}
\mathbf{E}(t) \cdot \frac{\partial \mathbf{D}(t)}{\partial t}=\frac{\omega_{0}}{2} \operatorname{Im}\left[\tilde{\mathbf{E}}\left(\omega_{0}\right) \cdot \overline{\tilde{\mathbf{D}}}\left(\omega_{0}\right) g^{2}(t)\right] \\
+\frac{d}{d t} \operatorname{Re}\left[\frac{1}{4} \tilde{\mathbf{E}}\left(\omega_{0}\right) \cdot \overline{\tilde{\mathbf{D}}}\left(\omega_{0}\right) g^{2}(t)\right] \\
+\frac{d}{d t} \operatorname{Re}\left[\frac{1}{4} \omega_{0} \tilde{\mathbf{E}}\left(\omega_{0}\right) \cdot \frac{\partial \overline{\mathbf{D}}\left(\omega_{0}\right)}{\partial \omega} g^{2}(t)-\frac{1}{4} \omega_{0} \overline{\tilde{\mathbf{D}}}\left(\omega_{0}\right) \cdot \frac{\partial \tilde{\mathbf{E}}\left(\omega_{0}\right)}{\partial \omega} g^{2}(t)\right] .
\end{array}
$$

Comparing (26) with (3), the stored electric field energy density and the rate of dissipated electric field energy density can easily be identified

$$
\begin{gathered}
w_{e}(t)=\frac{1}{4} \operatorname{Re}\left[\tilde{\mathbf{E}}\left(\omega_{0}\right) \cdot \overline{\tilde{\mathbf{D}}}\left(\omega_{0}\right) g^{2}(t)\right] \\
+\frac{1}{4} \omega_{0} \operatorname{Re}\left[\tilde{\tilde{\mathbf{E}}}\left(\omega_{0}\right) \cdot \frac{\partial \overline{\tilde{\mathbf{D}}}\left(\omega_{0}\right)}{\partial \omega} g^{2}(t)-\overline{\tilde{\mathbf{D}}}\left(\omega_{0}\right) \cdot \frac{\partial \tilde{\mathbf{E}}\left(\omega_{0}\right)}{\partial \omega} g^{2}(t)\right], \\
p_{e}(t)=\frac{\omega_{0}}{2} \operatorname{Im}\left[\tilde{\mathbf{E}}\left(\omega_{0}\right) \cdot \overline{\tilde{\mathbf{D}}}\left(\omega_{0}\right) g^{2}(t)\right] .
\end{gathered}
$$

Taking the time average of the above expressions over one period of the mid-band frequency $\omega_{0}$, and considering the following calculation

$$
\frac{1}{T_{0}} \int_{0}^{T_{0}} g^{2}(t) d t=\left(\frac{2 \Omega}{\pi}\right)^{2}
$$

the time-averaged stored electric field energy density (27) and the rate of dissipated electric field energy density (28) can be expressed in terms of the phasors as follows

$$
\begin{aligned}
w_{e}\left(\omega_{0}\right)= & \frac{1}{4} \operatorname{Re} \mathbf{E}\left(\omega_{0}\right) \cdot \overline{\mathbf{D}}\left(\omega_{0}\right) \\
& +\frac{1}{4} \omega_{0} \operatorname{Re}\left[\mathbf{E}\left(\omega_{0}\right) \cdot \frac{\partial \overline{\mathbf{D}}\left(\omega_{0}\right)}{\partial \omega}-\overline{\mathbf{D}}\left(\omega_{0}\right) \cdot \frac{\partial \mathbf{E}\left(\omega_{0}\right)}{\partial \omega}\right],
\end{aligned}
$$




$$
p_{e}\left(\omega_{0}\right)=\frac{\omega_{0}}{2} \operatorname{Im}\left[\mathbf{E}\left(\omega_{0}\right) \cdot \overline{\mathbf{D}}\left(\omega_{0}\right)\right]
$$

A similar discussion applies to $\mathbf{H} \cdot \partial \mathbf{B} / \partial t$, and the time-averaged stored magnetic field energy density and rate of dissipated magnetic field energy density are found to be

$$
\begin{aligned}
w_{m}\left(\omega_{0}\right)= & \frac{1}{4} \operatorname{Re} \mathbf{H}\left(\omega_{0}\right) \cdot \overline{\mathbf{B}}\left(\omega_{0}\right) \\
+ & \frac{1}{4} \omega_{0} \operatorname{Re}\left[\mathbf{H}\left(\omega_{0}\right) \cdot \frac{\partial \overline{\mathbf{B}}\left(\omega_{0}\right)}{\partial \omega}-\overline{\mathbf{B}}\left(\omega_{0}\right) \cdot \frac{\partial \mathbf{H}\left(\omega_{0}\right)}{\partial \omega}\right], \\
& p_{e d}\left(\mathbf{r}, \omega_{0}\right)=\frac{\omega_{0}}{2} \operatorname{Im}\left[\mathbf{H}\left(\mathbf{r}, \omega_{0}\right) \cdot \overline{\mathbf{B}}\left(\mathbf{r}, \omega_{0}\right)\right] .
\end{aligned}
$$

The energy expressions (29)-(32) do not explicitly contain medium parameters, and are therefore universally applicable. It can be shown that they reduce to those obtained in previous publications for various lossless media[2]-[23], and agree with those obtained in [1], in which a complex domain approach was used by replacing $j \omega$ with the complex frequency $s=\alpha+j \omega$. By use of the Cauchy-Riemann conditions and the field expansion for sufficiently small $\alpha$, the energy expressions can be determined through the time-domain Poynting theorem. The new NB approach developed in this article is physically more intuitive compared with the complex domain approach and may be thought of as a general technique for studying the timeharmonic field problems where an NB approximation in frequency domain must be assumed.

It is noted that all the field quantities in (29)-(32) are phasors for the sinusoidal field rather than the time-dependent complex envelopes in traditional NB analysis. A perplexing question that may arise is why the frequency derivatives occur in the stored energy expressions (29) and (31) for a pure sinusoidal field has only a single frequency component. One answer to the question is that all EM field quantities depend on frequency in microwave regime, and the frequency can thus be taken as a parameter and is allowed to vary with the purpose of examining how a field quantity changes with frequency. As a matter of fact, the frequency derivatives of field quantities (or circuit parameters) for a sinusoidal field appear frequently in the study of the stored field energy and group delay[25]-[27]. On the other hand, the new NB approach clearly reveals how the frequency derivatives of the fields show up from a different perspective. It indicates that the frequency derivatives of the fields are required essentially in order for the NB field to be accurately represented in the Fourier frequency domain.

\section{REFERENCES}

[1]. W. Geyi, "Stored electromagnetic field energies in general materials," $J$. Opt. Soc. Am. B, vol. 36, 917-925, 2019.

[2]. V. L. Ginzburg, The Propagation of Electromagnetic Waves in Plasmas, Pergman Press, 1964.

[3]. V. L. Ginzburg, Applications of Electrodynamics in Theoretical Physics and Astrophysics, Chapter 13, CRC Press, 1989.

[4]. J. D. Jackson, Classical Electrodynamics (3rd ed.), New York: John Wiley \& Sons, 258-267, 1998.

[5]. L. D. Landau, E. M. Lifshitz and L. P. Pitaevskii, Electrodynamics of Continuous Media (2nd ed)," Pergamon, Oxford, 274-276,1960.

[6]. L. B. Felsen and N. Marcuvitz, Radiation and Scattering of Waves, IEEE Press, 78-83,1994.

[7]. L. Brillouin, Wave Propagation and Group Velocity, Academic Press, 1960.
[8]. A. Tonning, "Energy density in continuous electromagnetic media," IEEE Trans. Antennas and Propagat. Vol.AP-8, 428-434,1960.

[9]. X. Zheng and P. Palffy-Muhoray, "Electrical energy storage and dissipation in materials," Phys. Lett. A, vol. 379, 1853-1856,2015.

[10]. J. Askne and B. Lind, "Energy of Electromagnetic Waves in the Presence of Absorption and Dispersion," Phys. Rev. A 2, 2335-2340,1970.

[11]. R. W. Ziolkowski, "Superluminal transmission of information through an electromagnetic medium," Phys. Rev. E 63, 046604,2001.

[12]. T. J. Cui and J. A. Kong, "Time-domain electromagnetic energy in a frequency-dispersive left-handed medium," Phys. Rev. B 70, 205106,2004.

[13]. A. D. Boardman and K. Marinov, "Electromagnetic energy in a dispersive metamaterial," Phys. Rev. B 73, 165110,2006.

[14]. P. G. Luan, "Power loss and electromagnetic energy density in a dispersive metamaterial medium," Phys. Rev. E 80, 046601,2009.

[15]. R. Ruppin, "Electromagnetic energy density in a dispersive and absorptive material," Phys. Lett. A, vol. 299, 309-312,2002.

[16]. O. B. Vorobyev, "Energy density of macroscopic electric and magnetic fields in dispersive medium with losses," Progress In Electromagnetics Research B, vol. 40, 343-360,2012.

[17]. R. Loudon, "The propagation of electromagnetic energy through an absorbing dielectric," J. Phys. A: General Physics, vol 3, No.4, 233-245,1970.

[18]. J. M. Carcione, "On energy definition in electromagnetism: An analogy with viscoelasticity," J. Acoust. Soc. Am. 105 (2), Pt. 1, 626-632,1999.

[19]. K. J. Webb and Shivanand, "Electromagnetic field energy in dispersive materials," J. Opt. Soc. Am. B, vol. 27, 1215-1220,2010.

[20]. F. D. Nunes, T. C. Vasconcelos, M. Bezerra, and J. Weiner, "Electromagnetic energy density in dispersive and dissipative media," $J$. Opt. Soc. Am. B, vol. 28,1544-1552,2011.

[21]. S. A. Tretyakov, "Electromagnetic field energy density in artificial microwave materials with strong dispersion and loss," Phys. Lett. A, vol. 343, 231-237,2005.

[22]. P. Ikonen and S. Tretyakov, "Determination of generalized permeability function and field energy density in artificial magnetics using the equivalent circuit model," IEEE Trans. Microw. Theory Tech., vol. 55, 92-99,2007.

[23]. P. C. W. Fung and K. Young, "Electric energy density in a dissipative medium by circuit analog," Am. J. Phys. 46, 57-59,1978.

[24]. S. O. Rice, "Envelopes of narrow-band signals," Proc. IEEE, vol. 70, 692-699, 1982.

[25]. C. G. Montgomery, R. H. Dicke and E. M. Purcell, Principles of Microwave Circuits, McGraw-Hill,151-156,1948.

[26]. H. J. Carlin, "Network theory without circuit elements," Proc. IEEE, 55, 482-497, 1967.

[27]. P. Penfield, Jr., R. Spencer and S. Duinker, Tellegen's Theorem and Electrical Networks, The MIT Press, 63-67,1970.

[28]. A. Papoulis, The Fourier Integral and Its Applications, McGraw-Hill Book Company, 20-21,1962. 